\title{
Hepatic Angiomyolipoma with a Giant Hemangioma
}

\author{
Aya Tani ${ }^{1}$, Hiroshi Yoshida ${ }^{1,2}$, Yasuhiro Mamada ${ }^{1}$, Nobuhiko Taniai ${ }^{1}$, \\ Sho Mineta ${ }^{1}$, Masato Yoshioka ${ }^{1}$, Yoichi Kawano ${ }^{1}$ Junji Ueda ${ }^{1}$, \\ Zenya Naito ${ }^{3}$ and Eiji Uchida ${ }^{1}$ \\ ${ }^{1}$ Surgery for Organ Function and Biological Regulation, Graduate School of Medicine, Nippon Medical School \\ ${ }^{2}$ Department of Surgery, Nippon Medical School Tama Nagayama Hospital \\ ${ }^{3}$ Department of Pathology, Nippon Medical School
}

\begin{abstract}
Hepatic angiomyolipoma is a rare hepatic mesenchymal tumor. We report a case of hepatic angiomyolipoma that was successfully resected along with a giant hemangioma. A 53year-old Japanese woman was admitted to our hospital for further evaluation of a liver tumor in segment 4. The tumor was detected on positron emission tomography during a health check-up. Abdominal ultrasonography revealed a well-defined mass of mixed echogenicity, 1.5 $\mathrm{cm}$ in diameter, in segment 4 , and a giant hemangioma of mixed echogenicity, $7 \mathrm{~cm}$ in diameter, in segment 7 . On enhanced computed tomography, the tumor in segment 4 showed hyperattenuation in the early phase and hypoattenuation in the delayed phase. On magnetic resonance imaging, the tumor in segment 4 showed hypointensity on T1-weighted images, hyperintensity on T2-weighted images, and hyperintensity on diffusion-weighted images. On angiography, the tumor in segment 4 appeared as a circumscribed hypervascular mass in the early phase and a slightly hypovascular mass in the delayed phase. The imaging findings suggested a primary hepatocellular carcinoma. The patient consented to resection of the tumor in segment 4 along with the giant hemangioma in segment 7 . These tumors were resected with tumor-free surgical margins by partial resection of segments 4 and 7 of the liver. The cut surface of the resected specimen of segment 4 showed a yellowish tumor consisting of mature adipose tissue. The histopathological diagnoses of the resected specimens were angiomyolipoma in segment 4 and cavernous hemangioma in segment 7 . The tumor in segment 4 consisted of mature lipocytes with angiomatous and small lymphocytic components, but no mitotic figures. The tumor showed immunoreactivity to smooth muscle antigen and homatropine methylbromide 45 and no immunoreactivity to AE/E3. The postoperative course was uneventful, and the patient remains well 1 year after the operation.
\end{abstract}

(J Nippon Med Sch 2011; 78: 317-321)

Key words: angiomyolipoma, liver, hemangioma, hepatectomy

Correspondence to Hiroshi Yoshida, MD, Department of Surgery, Nippon Medical School Tama Nagayama Hospital, 1-7-1 Nagayama, Tama, Tokyo 206-8512, Japan

E-mail: hiroshiy@nms.ac.jp

Journal Website (http://www.nms.ac.jp/jnms/) 


\section{Introduction}

Hepatic angiomyolipoma (HAML) is a rare hepatic mesenchymal tumor. Since Isak et al. ${ }^{1}$ reported the first case in 1976, more than 200 cases have been documented, especially in recent years ${ }^{2-4}$. HAML is composed of a heterogeneous mixture of adipose cells, smooth muscle cells, and vessels. HAML can be treated conservatively in the absence of spontaneous hemorrhage or malignant changes ${ }^{5,6}$. The radiologic features of HAML depend on the relative proportion of adipose cells ${ }^{4}$. Consequently, preoperative diagnosis of HAML is occasionally challenging. Differentiation from other liver tumors, especially hepatocellular carcinoma, can also be difficult. We report a case of HAML that was resected successfully along with a giant hemangioma.

\section{Case Report}

A 53-year-old Japanese woman was admitted to our hospital for further evaluation of a liver tumor in segment 4 . The tumor in segment 4 was detected on positron emission tomography (PET) during a health check-up. The tumor did not absorb fluorodeoxyglucose on PET. The patient had no history of liver disease or hepatitis and did not abuse alcohol. There was no abnormality on laboratory tests. Serum surface antigens for hepatitis $\mathrm{B}$ and anti-hepatitis $\mathrm{C}$ virus antibodies were negative. Tumor markers (e.g., $\alpha$-fetoprotein, PIVKA-2, carcinoembryonic antigen, and carbohydrate antigen 19-9) were negative. Abdominal ultrasonography revealed a well-defined mass of mixed echogenicity, $1.5 \mathrm{~cm}$ in diameter, in segment 4, and a giant hemangioma of mixed echogenicity, $7 \mathrm{~cm}$ in diameter, in segment 7 . On enhanced computed tomography (CT), the tumor in segment 4 showed hyperattenuation in the early phase and hypoattenuation in the delayed phase (Fig. 1a). A giant hemangioma was seen in segment 7 (Fig. 1b). On magnetic resonance imaging (MRI), the tumor in segment 4 showed hypointensity on T1-weighted images and hyperintensity on T2weighted images (Fig. 2). On angiography, the tumor
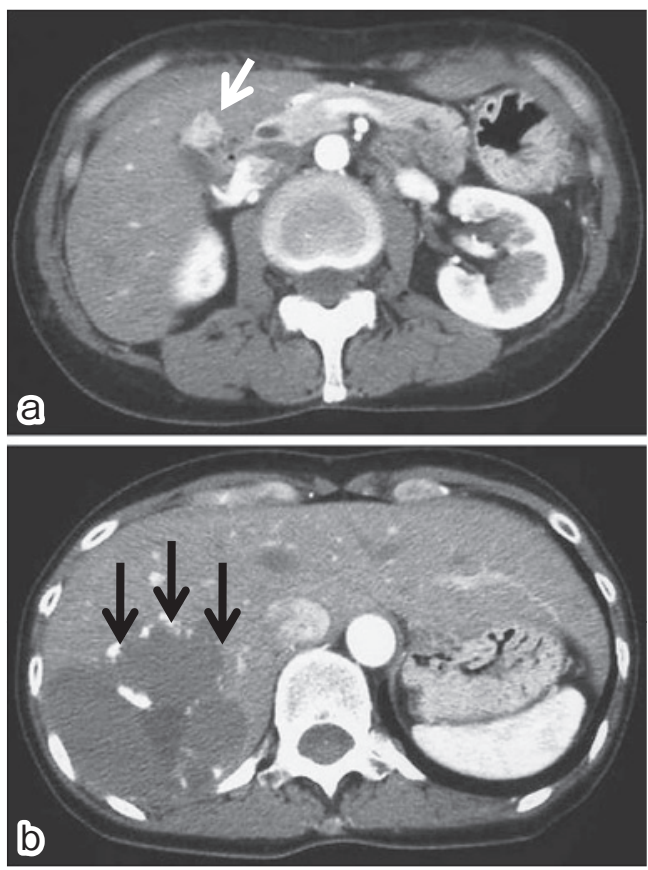

Fig. 1 On enhanced computed tomography, the tion in the early phase and hypoattenuation in the delayed phase (white arrow) (a). A giant hemangioma was seen in segment 7 (black arrows) (b).
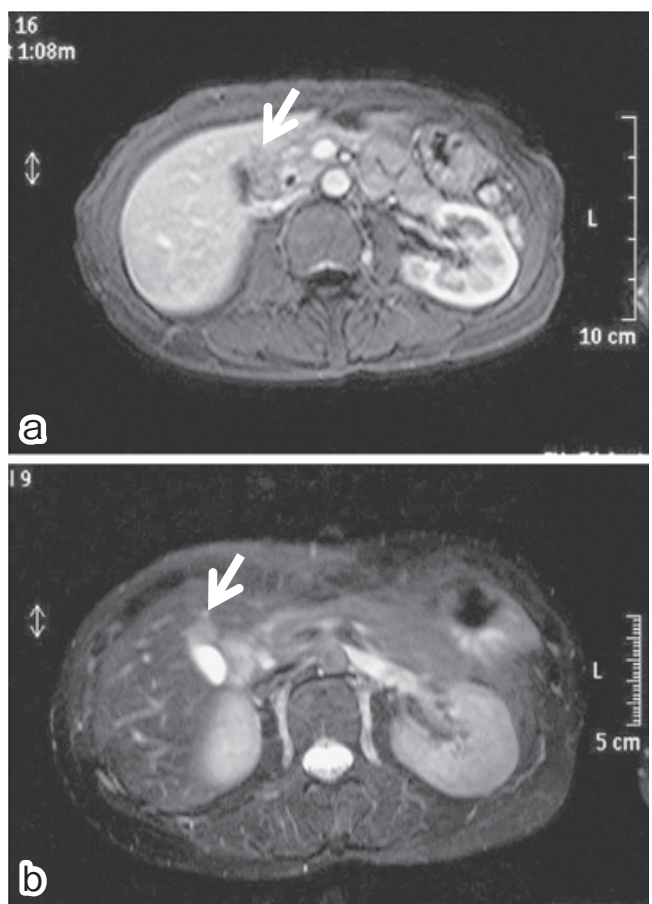

Fig. 2 On magnetic resonance imaging, the tumor in segment 4 showed hypointensity on T1weighted images (white arrow) (a) and hyperintensity on T2-weighted images (white arrow) (b). 


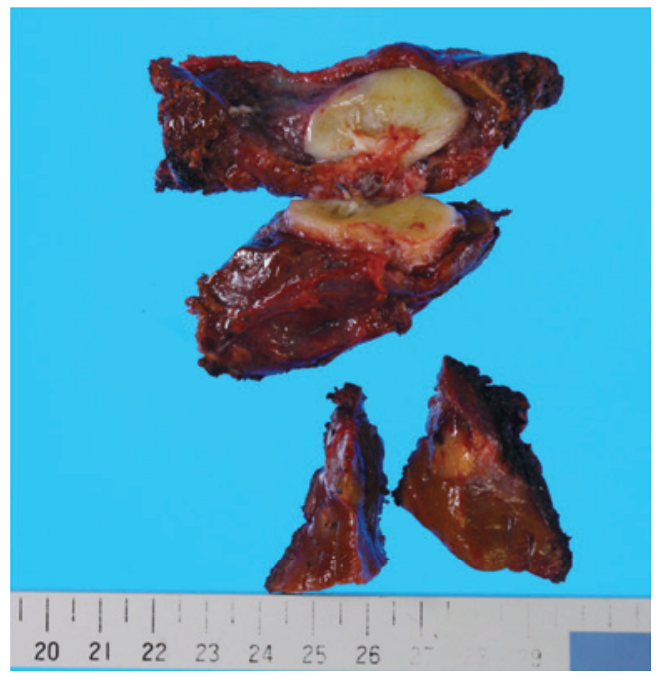

Fig. 3 The cut surface of the resected specimen of segment 4 showed a yellowish tumor consisting of mature adipose tissue.

in segment 4 appeared as a circumscribed hypervascular mass in the early phase and a slightly hypovascular mass in the delayed phase. The imaging findings suggested a primary hepatocellular carcinoma. Fine-needle aspiration biopsy was thought to be dangerous because the tumor in segment 4 was hypervascular and near the surface of the liver. After informed consent was obtained, the patient desired surgical treatment. The patient was scheduled to undergo resection of the tumor in segment 4 along with the giant hemangioma in segment 7 .

Laparotomy was performed, and a solid mass, 1.5 $\mathrm{cm}$ in diameter, in segment 4 and a soft mass, $7 \mathrm{~cm}$ in diameter, in segment 7 were detected. These tumors were resected with tumor-free surgical margins by partially resecting segments 4 and 7 of the liver. The cut surface of the resected specimen of segment 4 showed a yellowish tumor consisting of mature adipose tissue (Fig. 3). The clinical diagnosis of the tumor in the resected specimen of segment 7 was cavernous hemangioma. The histopathological diagnoses were angiomyolipoma in segment 4 and cavernous hemangioma in segment 7 . The nontumorous portion was composed of normal liver. The tumor in segment 4 was composed of mature lipocytes with angiomatous and small lymphocytic components but no mitotic figures. The tumor showed immunoreactivity to smooth muscle antigen
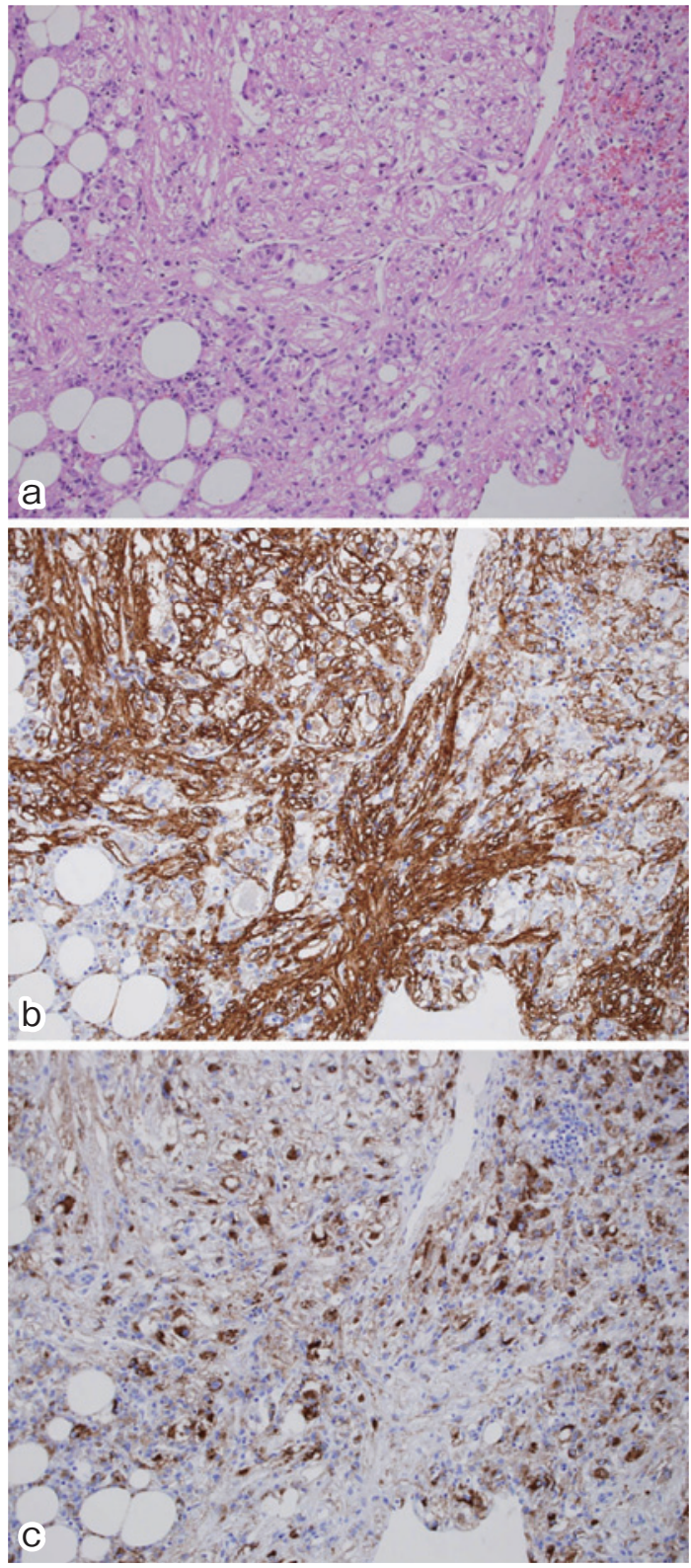

Fig. 4 On histopathological examination, the tumor in segment 4 was composed of mature lipocytes with angiomatous and small lymphocytic components but showed no mitotic figures (a) $(\mathrm{HE} \times 200)$. There was immunoreactivity to SMA (b) $(\times 200)$ and homatropine methylbromide 45 (c) $(\times 200)$.

(SMA) and homatropine methylbromide 45 (HMB45), but no immunoreactivity to AE/E3 (Fig. 4). The postoperative course was uneventful, and the patient remains well 1 year after the operation.

\section{Discussion}

Angiomyolipoma is a benign mesenchymal tumor, 
most commonly arising in the kidney. The liver is the second most common site of involvement, but HAML is much more rare than renal angiomyolipoma. HAML most often occurs in females and is usually solitary. The onset of disease is covert, and most cases lack clinically significant symptoms. The results of laboratory tests are usually normal, and there is no specific tumor marker for HAML. Most patients have no history of viral hepatitis.

Nonomura et $\mathrm{al}^{4}$ have reported that typical imaging features of HAML include: 1) high heterogeneous echoes on ultrasonography; 2) mixed low density (CT value $<20$ Hounsfield units) on CT images; 3) high signal intensity on T1- and T2weighted MRI; and 4) rich vessels and tumor staining on angiography. A comprehensive evaluation of findings on ultrasonography, CT, MRI, and angiography may enhance the accuracy of preoperative diagnosis, but different contents and distributions of vessels, smooth muscle, and fat in particular (varying from $5 \%$ to $90 \%)^{7}$ can result in diverse imaging profiles of HAML. All of these factors make preoperative diagnosis difficult. Nearly half of all HAMLs have been first misdiagnosed as carcinomas or sarcomas ${ }^{22}$. Thus, it is essential to recognize the pitfalls of HAML diagnosis. If lesions have detectable fat and vascular structures, the diagnosis of HAML is straightforward. The most difficult problem in diagnosis is atypical presentations (including necrosis, hemorrhage in tumors, or myomatous-type tumors $)^{4.8}$, which can create difficulty in distinguishing HAML from other hepatic tumors.

Histologically, HAML consists of a heterogeneous mixture of blood vessels, smooth muscle, and adipose cells, varying in proportions and distributions not only among different tumors, but from area to area within the same tumor. This heterogeneity can result in misdiagnosis of HAML as a hepatocellular carcinoma or other type of liver tumor. On the basis of the predominant component, HAML can be categorized into several subclasses, including mixed type (the most common type), lipomatous (more than $70 \%$ fat), myomatous (less than $10 \%$ fat), and angiomatous. Awareness of broad differences in the proportion of fat can facilitate the diagnosis of fatcontaining tumors of the liver. In the present case, the tumor in segment 4 consisted of mature lipocytes with angiomatous and small lymphocytic components but no mitotic figures. Several studies have reported that HMB45, a human monoclonal antibody to melanocytes, is consistently positive in angiomyolipomas ${ }^{9,10}$. Ren et al. $^{11}$ have reported that HMB45 immunostaining was positive in all 26 cases of HAML. Sturtz and Dabbs ${ }^{9}$ have reported 15 cases of angiomyolipoma that showed positive immunoreactivity to HMB45. Therefore, HMB45 has become a definitive tool for the diagnosis of HAML. Other markers, such as S100 and SMA, may also be helpful. In our patient, the tumor in segment 4 was immunoreactive for SMA and HMB45. Li et al. ${ }^{12}$ have reported that CD117 is positive in only $25 \%$ of lesions, making further investigations essential. The origin of HAML tissue remains unclear. HAML is considered a mesenchymal hamartoma, while recent studies have shown that it originates from perivascular epithelioid cells, which can multidirectionally differentiate into vascular smooth muscle and epithelial cells, characterized by the expression of melanoma cell differentiationassociated markers ${ }^{3}$.

Most cases of HAML have a good prognosis regardless of the management strategy, including hepatic resection or conservative treatment (observation) after echo-guided needle biopsy ${ }^{13}$. Several authors have suggested that HAML can be managed conservatively with follow-up after fineneedle aspiration biopsy ${ }^{8,14,15}$. Surgical intervention may be needed in selected cases to alleviate the mass effect on neighboring organs ${ }^{3,13}$. The major risks of conservative management are spontaneous rupture and malignant transformation of the mass $^{3,16-18}$. In fact, malignant HAML was first reported in $2000^{18}$. Because of these risks, surgical resection has also been recommended as the treatment of choice ${ }^{16,17}$. However, in a previous series, one patient died of congestive heart failure after surgery, and another died of recurrent disease 14 months after surgery ${ }^{16,17}$. These findings illustrate the potential risks of surgical management.

Hemangiomas are the most common primary liver 
tumor. The size of most hepatic hemangiomas remains stable ${ }^{1920}$. A giant hemangioma, defined as a hemangioma exceeding $4 \mathrm{~cm}$ in diameter, can cause symptoms and require treatment ${ }^{21}$. Complications of hemangiomas include congestion, bleeding, thrombosis, infarction, Kasabach-Merritt syndrome, spontaneous rupture, obstructive jaundice, and gastric outlet obstruction ${ }^{21-26}$. In our patient, a liver tumor was found on PET during a health check-up. Imaging findings suggested a primary hepatocellular carcinoma. After informed consent was obtained, the patient underwent resection of the tumor along with the giant hemangioma. A diagnosis of HAML was confirmed histologically. The patient has remained well since the operation, indicating that surgery was an effective treatment strategy for HAML.

\section{References}

1. Isak K: Mesenchymal tumors of the liver. In Hepatocellular carcinoma (Okuda K, Peters R, eds), 1976; pp 247-307, John Wiley \& Sons, New York.

2. Tsui WM, Colombari R, Portmann BC, et al.: Hepatic angiomyolipoma: a clinicopathologic study of 30 cases and delineation of unusual morphologic variants. Am J Surg Pathol 1999; 23: 34-48.

3. Yang CY, Ho MC, Jeng YM, Hu RH, Wu YM, Lee PH: Management of hepatic angiomyolipoma. J Gastrointest Surg 2007; 11: 452-457.

4. Nonomura A, Mizukami Y, Kadoya M: Angiomyolipoma of the liver: a collective review. J Gastroenterol 1994; 29: 95-105.

5. Guidi G, Catalano O, Rotondo A: Spontaneous rupture of a hepatic angiomyolipoma: CT findings and literature review. Eur Radiol 1997; 7: 335-337.

6. Takayama Y, Moriura S, Nagata J, et al.: Hepatic angiomyolipoma: radiologic and histopathologic correlation. Abdom Imaging 2002; 27: 180-183.

7. Goodman ZD, Ishak KG: Angiomyolipomas of the liver. Am J Surg Pathol 1984; 8: 745-750.

8. Cha I, Cartwright D, Guis M, Miller TR, Ferrell LD: Angiomyolipoma of the liver in fine-needle aspiration biopsies: its distinction from hepatocellular carcinoma. Cancer 1999; 87: 25-30.

9. Sturtz CL, Dabbs DJ: Angiomyolipomas: the nature and expression of the HMB45 antigen. Mod Pathol 1994; 7: 842-845.

10. Terris B, Flejou JF, Picot R, Belghiti J, Henin D: Hepatic angiomyolipoma. A report of four cases with immunohistochemical and DNA-flow cytometric studies. Arch Pathol Lab Med 1996; 120: 68-72.

11. Ren N, Qin LX, Tang ZY, Wu ZQ, Fan J: Diagnosis and treatment of hepatic angiomyolipoma in 26 cases. World J Gastroenterol 2003; 9: 1856-1858.

12. Li $\mathrm{T}$, Wang $\mathrm{L}, \mathrm{Yu} \mathrm{HH}$, et al.: Hepatic angiomyolipoma: a retrospective study of 25 cases. Surg Today 2008; 38: 529-535.

13. Yeh CN, Chen MF, Hung CF, Chen TC, Chao TC: Angiomyolipoma of the liver. J Surg Oncol 2001; 77: 195-200.

14. Ma TK, Tse MK, Tsui WM, Yuen KT: Fine needle aspiration diagnosis of angiomyolipoma of the liver using a cell block with immunohistochemical study. A case report. Acta Cytol 1994; 38: 257-260.

15. Irie $H$, Honda $H$, Kuroiwa $T$, et al.: Hepatic angiomyolipoma: report of changing size and internal composition on follow-up examination in two cases. J Comput Assist Tomogr 1999; 23: 310-313.

16. $\mathrm{Xu} \mathrm{L}$, Shao $\mathrm{Y}$, Zhang $\mathrm{H}$, Wang $\mathrm{H}$ : Hepatic angiomyolipoma: report of 8 cases and review of literature. Asian J Surg 2002; 25: 82-86; discussion 78.

17. Zhou YM, Li B, Xu F, et al.: Clinical features of hepatic angiomyolipoma. Hepatobiliary Pancreat Dis Int 2008; 7: 284-287.

18. Dalle I, Sciot R, de Vos R, et al.: Malignant angiomyolipoma of the liver: a hitherto unreported variant. Histopathology 2000; 36: 443-450.

19. Weimann A, Ringe B, Klempnauer J, et al: Benign liver tumors: differential diagnosis and indications for surgery. World J Surg 1997; 21: 983-990; discussion 90-91.

20. Yamagata M, Kanematsu T, Matsumata $T$, Utsunomiya T, Ikeda Y, Sugimachi K: Management of haemangioma of the liver: comparison of results between surgery and observation. Br J Surg 1991; 78: 1223-1225.

21. Adam YG, Huvos AG, Fortner JG: Giant hemangiomas of the liver. Ann Surg 1970; 172: 239245.

22. Srivastava DN, Gandhi D, Seith A, Pande GK, Sahni P: Transcatheter arterial embolization in the treatment of symptomatic cavernous hemangiomas of the liver: a prospective study. Abdom Imaging 2001; 26: 510-514.

23. Herman P, Costa ML, Machado MA, et al.: Management of hepatic hemangiomas: a 14-year experience. J Gastrointest Surg 2005; 9: 853-859.

24. Farges O, Daradkeh S, Bismuth H: Cavernous hemangiomas of the liver: are there any indications for resection? World J Surg 1995; 19: 19-24.

25. Belli L, De Carlis L, Beati C, Rondinara G, Sansalone V, Brambilla G: Surgical treatment of symptomatic giant hemangiomas of the liver. Surg Gynecol Obstet 1992; 174: 474-478.

26. Iwatsuki S, Todo S, Starzl TE: Excisional therapy for benign hepatic lesions. Surg Gynecol Obstet 1990; 171: $240-246$

(Received, March 29, 2011)

(Accepted, May 6, 2011) 